\title{
Ramsey numbers and adiabatic quantum computing
}

\author{
Frank Gaitan ${ }^{1}$ and Lane Clark $^{2}$ \\ ${ }^{1}$ Laboratory for Physical Sciences, 8050 Greenmead Dr, College Park, MD 20740 \\ ${ }^{2}$ Department of Mathematics, Southern Illinois University, Carbondale, IL 62901-4401
}

(Dated: October 14, 2018)

\begin{abstract}
The graph-theoretic Ramsey numbers are notoriously difficult to calculate. In fact, for the twocolor Ramsey numbers $R(m, n)$ with $m, n \geq 3$, only nine are currently known. We present a quantum algorithm for the computation of the Ramsey numbers $R(m, n)$. We show how the computation of $R(m, n)$ can be mapped to a combinatorial optimization problem whose solution can be found using adiabatic quantum evolution. We numerically simulate this adiabatic quantum algorithm and show that it correctly determines the Ramsey numbers $R(3,3)$ and $R(2, s)$ for $5 \leq s \leq 7$. We then discuss the algorithm's experimental implementation, and close by showing that Ramsey number computation belongs to the quantum complexity class QMA.
\end{abstract}

PACS numbers: 03.67.Ac,02.10.Ox,89.75.Hc

In an arbitrary party of $N$ people one might ask whether there is a group of $m$ people who are all mutually acquainted, or a group of $n$ people who are all mutual strangers. Using Ramsey theory [1, 2], it can be shown that a threshold value $R(m, n)$ exists for the party size $N$ so that when $N \geq R(m, n)$, all parties of $N$ people will either contain $m$ mutual acquaintances, or $n$ mutual strangers. The threshold value $R(m, n)$ is an example of a two-color Ramsey number. Other types of Ramsey numbers exist, though we will focus on two color Ramsey numbers in this paper.

One can represent the $N$-person party problem by an $N$-vertex graph. Here each person is associated with a vertex, and an edge is drawn between a pair of vertices only when the corresponding people know each other. In the case where $m$ people are mutual acquaintances, there will be an edge connecting any pair of the $m$ corresponding vertices. Similarly, if $n$ people are mutual strangers, there will be no edge between any of the $n$ corresponding vertices. In the language of graph theory [3] the $m$ vertices form an $m$-clique, and the $n$ vertices form an $n$ independent set. The party problem is now a statement in graph theory: if $N \geq R(m, n)$, every graph with $N$ vertices will contain either an $m$-clique, or an $n$-independent set. Ramsey numbers can also be introduced using colorings of complete graphs, and $R(m, n)$ corresponds to the case where only two colors are used.

Ramsey theory has found applications in mathematics, information theory, and theoretical computer science [6]. An application of fundamental significance appears in the Paris-Harrington $(\mathrm{PH})$ theorem of mathematical logic [4] which established that a particular statement in Ramsey theory related to graph colorings and natural numbers is true, though unprovable within the axioms of Peano arithmetic. Such statements are known to exist as a consequence of Godel's incompleteness theorem, though the $\mathrm{PH}$ theorem provided the first natural example. Deep connections have also been shown to exist between Ramsey theory, topological dynamics, and ergodic theory [5].
Ramsey numbers grow extremely quickly and so are notoriously difficult to calculate. In fact, for two color Ramsey numbers $R(m, n)$ with $m, n \geq 3$, only nine are presently known [3]. To check whether $N \stackrel{?}{=} R(m, n)$ requires examining all $2^{N(N-1) / 2} N$-vertex graphs. The number of graphs to be checked thus grows superexponentially with $N$, and so the task quickly becomes intractable. Ketonen and Solovay [7] have shown that this is the root cause for why the statement in the $\mathrm{PH}$ theorem cannot be proved within Peano arithmetic.

In this paper we: (i) present a quantum algorithm for calculating Ramsey numbers based on adiabatic quantum evolution; (ii) numerically simulate the algorithm to verify that it correctly calculates small Ramsey numbers; (iii) discuss its experimental implementation; and (iv) show that Ramsey number computation belongs to the quantum complexity class $Q M A$.

Optimization Problem: We begin by establishing a 1-1 correspondence between the set of $N$-vertex graphs and binary strings of length $L=N(N-1) / 2$. To each $N$ vertex graph $G$ there corresponds a unique adjacency matrix $A(G)$ which is an $N \times N$ symmetric matrix with vanishing diagonal matrix elements, and with off-diagonal element $a_{i, j}=1(0)$ when distinct vertices $i$ and $j$ are (are not) joined by an edge. It follows that $A(G)$ is determined by its lower triangular part. By concatenating column-wise the matrix elements $a_{i, j}$ appearing below the principal diagonal, we can construct a unique binary string $g(G)$ of length $L$ for each graph $G$ :

$$
g(G) \equiv a_{2,1} \cdots a_{N, 1} a_{3,2} \cdots a_{N, 2} \cdots a_{N, N-1} .
$$

Given the string $g(G)$, the following procedure determines the number of $m$-cliques in $G$. Choose $m$ vertices $S_{\alpha}=\left\{v_{1}, \ldots, v_{m}\right\}$ from the $N$ vertices of $G$ and form the product $\mathcal{C}_{\alpha}=\prod_{\left(v_{j}, v_{k} \in S_{\alpha}\right)}^{(j \neq k)} a_{v_{j}, v_{k}}$. Note that $\mathcal{C}_{\alpha}=1$ when $S_{\alpha}$ forms an $m$-clique; otherwise $\mathcal{C}_{\alpha}=0$. Now repeat this procedure for all $\rho=C(N, m)$ ways of choosing $m$ vertices from $N$ vertices, and form the sum $\mathcal{C}(G)=$ 
$\sum_{\alpha=1}^{\rho} \mathcal{C}_{\alpha}$. By construction, $\mathcal{C}(G)$ equals the number of $m$-cliques contained in $G$. A similar procedure determines the number of $n$-independent sets in $G$. Briefly, choose $n$ vertices $T_{\alpha}=\left\{v_{1}, \ldots, v_{n}\right\}$ from the $N$ vertices in $G$, and form the product $\mathcal{I}_{\alpha}=\prod_{\left(v_{j}, v_{k} \in T_{\alpha}\right)}^{(j \neq k)} \bar{a}_{v_{j}, v_{k}}$, where $\bar{a}_{v_{j}, v_{k}}=1-a_{v_{j}, v_{k}}$. If the vertex set $T_{\alpha}$ forms an $n$ independent set, then $\mathcal{I}_{\alpha}=1$; otherwise $\mathcal{I}_{\alpha}=0$. Repeat this for all $\nu=C(N, n)$ ways of choosing $n$ vertices from $N$ vertices, then form the sum $\mathcal{I}(G)=\sum_{\alpha=1}^{\nu} \mathcal{I}_{\alpha}$. By construction, $\mathcal{I}(G)$ gives the number of $n$-independent sets contained in $G$. Finally, define

$$
h(G)=\mathcal{C}(G)+\mathcal{I}(G) .
$$

It follows from the above discussion that $h(G)$ is the total number of $m$-cliques and $n$-independent sets in $G$. Thus $h(G) \geq 0$ for all graphs $G$; and $h(G)=0$ if and only if $G$ does not contain an $m$-clique or $n$-independent set.

We can use $h(G)$ as the cost function for the following combinatorial optimization problem. For given integers $(N, m, n)$, and with $h(G)$ defined as above, find an $N$-vertex graph $G_{*}$ that yields the global minimum of $h(G)$. Notice that if $N<R(m, n)$, the (global) minimum will be $h\left(G_{*}\right)=0$ since Ramsey theory guarantees that a graph exists which has no $m$-clique or $n$-independent set. On the other hand, if $N \geq R(m, n)$, Ramsey theory guarantees $h\left(G_{*}\right)>0$. If we begin with $N<R(m, n)$ and increment $N$ by 1 until we first find $h\left(G_{*}\right)>0$, then the corresponding $N$ will be exactly $R(m, n)$. We now show how this combinatorial optimization problem can be solved using adiabatic quantum evolution.

Quantum Algorithm: The adiabatic quantum evolution (AQE) algorithm [8] exploits the adiabatic dynamics of a quantum system to solve combinatorial optimization problems. The AQE algorithm uses the optimization problem cost function to define a problem Hamiltonian $H_{P}$ whose ground-state subspace encodes all problem solutions. The algorithm evolves the state of an $L$-qubit register from the ground-state of an initial Hamiltonian $H_{i}$ to the ground-state of $H_{P}$ with probability approaching 1 in the adiabatic limit. An appropriate measurement at the end of the adiabatic evolution yields a solution of the optimization problem almost certainly. The timedependent Hamiltonian $H(t)$ for global AQE is

$$
H(t)=\left(1-\frac{t}{T}\right) H_{i}+\left(\frac{t}{T}\right) H_{P},
$$

where $T$ is the algorithm runtime, and adiabatic dynamics corresponds to $T \rightarrow \infty$.

To map the optimization problem associated with computing $R(m, n)$ onto an adiabatic quantum computation, we begin with the 1-1 correspondence between $N$-vertex graphs $G$ and length $L=N(N-1) / 2$ binary strings $g(G)$. From Eq. (1) we see that position along the string is indexed by vertex pairs $(i, j)$. We thus identify a qubit with each such pair $(i, j)$, and will thus need $L$ qubits.
Defining the computational basis states (CBS) to be the eigenstates of $\sigma_{z}^{0} \otimes \cdots \otimes \sigma_{z}^{L-1}$, we identify the $2^{L}$ graph strings $g(G)$ with the $2^{L}$ CBS: $g(G) \rightarrow|g(G)\rangle$. The problem Hamiltonian $H_{P}$ is defined to be diagonal in the computational basis with eigenvalue $h(G)$ associated with eigenstate $|g(G)\rangle$ :

$$
H_{P}|g(G)\rangle=h(G)|g(G)\rangle .
$$

Note that the ground-state energy of $H_{P}$ will be zero iff there is a graph with no $m$-cliques or $n$-independent sets. We give an operator expression for $H_{P}$ below. The initial Hamiltonian $H_{i}$ is chosen to be

$$
H_{i}=\sum_{l=0}^{L-1} \frac{1}{2}\left(I^{l}-\sigma_{x}^{l}\right),
$$

where $I^{l}$ and $\sigma_{x}^{l}$ are the identity and x-Pauli operator for qubit $l$, respectively. The ground-state of $H_{i}$ is the easily constructed uniform superposition of CBS.

The quantum algorithm for computing $R(m, n)$ begins by setting $N$ equal to a strict lower bound for $R(m, n)$ which can be found using the probabilistic method [9] or a table of two-color Ramsey numbers [3]. The AQE algorithm is run on $L_{N}=N(N-1) / 2$ qubits, and the energy $E$ is measured at the end of algorithm execution. In the adiabatic limit the result will be $E=0$ since $N<R(m, n)$. The value of $N$ is now incremented $N \rightarrow N+1$, the AQE algorithm is re-run on $L_{N+1}$ qubits, and the energy $E$ measured at the end of algorithm execution. This process is repeated until $E>0$ first occurs, at which point the associated $N$ will be equal to $R(m, n)$. Note that any real application of AQE will only be approximately adiabatic. Thus the probability that the measured energy $E$ will be the ground-state energy will be $1-\epsilon$. In this case, the algorithm must be run $k \sim \mathcal{O}(\ln [1-\delta] / \ln \epsilon)$ times so that, with probability $\delta>1-\epsilon$, at least one of the measurement outcomes will be the true ground-state energy. We can make $\delta$ arbitrarily close to 1 by choosing $k$ sufficiently large.

Simulation Results: To test the adiabatic quantum computation of $R(m, n)$, we numerically simulated the Schrodinger dynamics generated by the AQE Hamiltonian $H(t)$. Clearly, these simulations can only be run at finite values of $T$. As in Ref. [10], we chose $T$ so that the algorithm success probability $P_{s}$ is large compared to the probability that a randomly chosen CBS will belong to the $D$-degenerate ground-state eigenspace of $H_{P}$ $\left.\left(P_{s} \gg D / 2^{L}\right)\right)$. Here $P_{s}$ is the probability that an energy measurement done at the final time $T$ will yield the ground-state energy $E_{g s}$ of $H_{P}$. Since a classical computer cannot efficiently simulate the dynamics of a quantum system, we can only obtain small Ramsey numbers. In this case, $H_{P}$ can be found by evaluating the cost function $h(G)$ using the procedure described above Eq. (2).

We simulated the AQE computation of $R(3,3)$ and $R(2, s)$ for $5 \leq s \leq 7$. Straightforward arguments [3] give 
$R(3,3)=6$ and $R(2, s)=s$. We present our simulation results in Table I. We see that for all $m, n$ considered, the threshold value $N_{t}$ where $E_{g s}>0$ first occurs is precisely at the Ramsey number: $N_{t}=R(m, n)$.

For $R(2, s)$ and $N=s$, Table $\llbracket$ gives $E_{g s}=1$. For these cases, graphs corresponding to ground-states of $H_{P}$ will thus contain either a single $s$-independent set or a single 2-clique. There is only one $s$-vertex graph with an $s$-independent set, and there are $C(s, 2)=s(s-1) / 2$ graphs with one 2-clique (viz. edge). Thus the groundstate degeneracy $D=1+C(s, 2)$, in agreement with the $R(2, s)$ degeneracies in Table \ for $N=s=5,6,7$. For $R(3,3)$ and $N=6$, Table \ gives $E_{g s}=2$. Thus graphs corresponding to ground-states are those with: (i) two 3cliques; (ii) two 3-independent sets; or (iii) one 3-clique and one 3-independent set. Ref. [11] derived the minimum number of 3-cliques and 3-independent sets that can be present in an $N$-vertex graph. This minimum is precisely our $E_{g s}$ for $R(3,3)$ and a given $N$. For $N=6$, the minimum value is 2 , in agreement with $E_{g s}=2$ in Table I. We carried out both analytical [12] and numerical counts of the ground-state graphs for $R(3,3)$ and $N=6$. Both approaches found 1760 graphs giving a ground-state degeneracy $D=1760$. In all cases appearing in Table @ the upward jump in $D$ seen upon reaching the Ramsey threshold $N=R(m, n)$ (from below) is responsible for the jump in the success probability $P_{s}$ also seen at this threshold.

Although we would like to have calculated larger Ramsey numbers, this was simply not practical. Note that the $N=7$ simulations use $L=21$ qubits. These simulations are at the upper limit of 20-22 qubits at which simulation of the full AQE Schrodinger dynamics is practical [10, 13, 14]. The next smallest Ramsey number is $R(2,8)=8$ which requires a 28 qubit simulation, well beyond what can be done practically.

Experimental Implementation: We begin by determining an operator expression for the problem Hamiltonian $H_{P}$ which then fixes the AQE Hamiltonian $H(t)$ through Eqs. (3) and (5). Recall that the eigenvalue $h(G)=\mathcal{C}(G)+\mathcal{I}(G)$ counts the total number of $m$ cliques and $n$-independent sets in a graph $G$. For an $m$-vertex set $S_{\alpha}=\left\{v_{1}, \ldots, v_{m}\right\}$, we define the edge set $E_{\alpha}=\left\{e_{k}^{\alpha}: k=1, \ldots, C(m, 2)\right\}$ as the set of all edges connecting pairs of vertices $v_{i}, v_{j} \in S_{\alpha}$, and $C(m, 2)$ is the number of ways of choosing 2 vertices out of $m$. If $S_{\alpha}$ corresponds to an $m$-clique in the graph $G$, the graph-string $g(G)$ must have 1's at all bit-positions associated with the edges of $E_{\alpha}$. Let the states $|0\rangle$ and $|1\rangle$ satisfy $\sigma_{z}|a\rangle=$ $(-1)^{a}|a\rangle$. Then the operator $H_{\alpha}=\prod_{e \in E_{\alpha}} P_{1}^{e}$ (where $P_{1}^{e}=(1 / 2)\left[I^{e}-\sigma_{z}^{e}\right]$, and $e$ labels the qubit associated with edge $e$ ) will have $|g(G)\rangle$ as an eigenstate with eigenvalue 1 when $S_{\alpha}$ is an $m$-clique, and zero otherwise. The operator that counts all $m$-cliques in a graph $G$ is then $H_{c l}^{m}=\sum_{\alpha=1}^{C(N, m)} H_{\alpha}$, and by construction, $H_{c l}^{m}|g(G)\rangle=$ $\mathcal{C}(G)|g(G)\rangle$. A similar analysis can be carried out for $n$ - independent sets. Let $T_{\alpha}=\left\{v_{1}, \ldots, v_{n}\right\}$ be an arbitrary $n$-vertex set, and $\bar{E}_{\alpha}$ its corresponding edge set. If $T_{\alpha}$ is an $n$-independent set in a graph $G$, then the graphstring $g(G)$ must have 0's at all bit-positions associated with the edges of $\bar{E}_{\alpha}$. The operator $\bar{H}_{\alpha}=\prod_{e \in \bar{E}_{\alpha}} P_{0}^{e}$ (where $P_{0}^{e}=(1 / 2)\left[I^{e}+\sigma_{z}^{e}\right]$, and $e$ labels the qubit associated with edge $e$ ) will have eigenstate $|g(G)\rangle$ with eigenvalue $1(0)$ when $T_{\alpha}$ is (is not) an $n$-independent set. The operator that counts all $n$-independent sets in an arbitrary graph $G$ is then $H_{i s}^{n}=\sum_{\alpha=1}^{C(N, n)} \bar{H}_{\alpha}$, and by construction, $H_{i s}^{n}|g(G)\rangle=\mathcal{I}(G)|g(G)\rangle$. For calculation of $R(m, n)$, the problem Hamiltonian $H_{P}^{N m n}$ is then

$$
H_{P}^{N m n}=H_{c l}^{m}+H_{i s}^{n} .
$$

Note that $H_{P}^{N m n}$ contains $\mathcal{O}\left(N^{s}\right)$ terms, where $N$ is the number of vertices and $s=\max \{C(N, m), C(N, n)\}$. Since each $H_{\alpha}$ and $\bar{H}_{\alpha}$ is a projection operator, their operator norm will be unity and their matrix elements, being 0's and 1's, are specified with a single bit. Lastly, note that each term in $H_{P}^{N m n}$ is a product of at most $t=\max \{C(m, 2), C(n, 2)\} \sigma_{z}$-operators so that $H_{P}^{N m n}$ is a $t$-local Hamiltonian [15]. By using perturbative gadgets, it can be reduced to a 2-local Hamiltonian [16 18 .

For a given Hamiltonian $H(t)$, two approaches have been demonstrated to experimentally implement AQE [19 21]. Refs. 19], 20] partitioned the full evolution into $\mathcal{N}$ subintervals of duration $\Delta t=T / \mathcal{N}$ which are sufficently short that the propagator $U_{l}$ for each subinterval $l$ can be factored via a Trotter expansion. This approach was applied to three-qubit systems, though it can be used for arbitrary size qubit systems. Ref. [21] describes experiments using a quantum annealing device designed to implement adiabatic quantum optimization algorithms. Results are reported of AQE solution for the groundstate of randomly generated instances of an 8-qubit quantum Ising spin glass. Work using perturbative gadgets is underway to convert $H_{P}^{N m n}$ into a 2-local form amenable to both AQE experimental approaches.

Ramsey Numbers and QMA: Quantum complexity theory formalizes the notion of efficient quantum algorithms. Our interest is in the quantum complexity class $Q M A$ which generalizes the randomized version of the classical complexity class $N P[15,16]$.

$Q M A$ is a class of promise problems where each problem $L$ is the union of two disjoint sets of binary strings $L_{y}$ and $L_{n}$ corresponding to Yes and No instances of the problem. For a string $x \in L_{y} \cup L_{n}$, the task is to determine whether $x \in L_{y}$ or $x \in L_{n}$ using polynomial resources. Let $\mathcal{H}$ denote a two-dimensional Hilbert space; and $|x\rangle$ the CBS labeled by the binary string $x$.

Definition 1 (QMA) Let $x \in L=L_{y} \cup L_{n}$ and $\epsilon=2^{-\Omega(|x|)}$. The promise problem $L$ belongs to QMA if there exists a quantum polynomial-time verifier $V(|x\rangle,|y\rangle) \rightarrow\{0,1\}$, and a polynomial $\pi(|x|)$ such that: (i) for all $x \in L_{y}$, there exists an $|\xi\rangle \in \mathcal{H}^{\pi(|x|)}$ 
TABLE I: Simulation results for Ramsey numbers $R(3,3)$ and $R(2, s)$ for $5 \leq s \leq 7$. Here $N$ is the number of graph vertices; $E_{g s}$ and $D$ are the ground-state energy and degeneracy, respectively, for the problem Hamiltonian $H_{P}$; and $T$ and $P_{s}$ are, respectively, the algorithm runtime and success probability.

\begin{tabular}{|c|c|c|c|c|c|c|c|c|c|c|c|c|c|c|c|c|c|c|c|}
\hline \multicolumn{5}{|c|}{$\mathbf{R}(2,5)$} & \multicolumn{5}{|c|}{$\mathrm{R}(2,6)$} & \multicolumn{5}{|c|}{$\mathbf{R}(\mathbf{3}, \mathbf{3})$} & \multicolumn{5}{|c|}{$\mathbf{R}(\mathbf{2}, \mathbf{7})$} \\
\hline$N$ & $E_{g s}$ & $D$ & $T$ & $P_{s}$ & $N$ & $E_{g s}$ & $D$ & $T$ & $P_{s}$ & $N$ & $E_{g s}$ & $D$ & $T$ & $P_{s}$ & $N$ & $E_{g s}$ & $D$ & $T$ & $P_{s}$ \\
\hline 3 & 0.0 & 1 & 5.0 & 0.591 & 4 & 0.0 & 1 & 5.0 & 0.349 & 4 & 0.0 & 18 & 5.0 & 0.769 & 5 & 0.0 & 1 & 8.0 & 0.865 \\
\hline 4 & 0.0 & 1 & 5.0 & 0.349 & 5 & 0.0 & 1 & 5.0 & 0.173 & 5 & 0.0 & 12 & 5.0 & 0.194 & 6 & 0.0 & 1 & 8.0 & 0.805 \\
\hline 5 & 1.0 & 11 & 5.0 & 0.518 & 6 & 1.0 & 16 & 5.0 & 0.286 & 6 & 2.0 & 1760 & 5.0 & 0.693 & 7 & 1.0 & 22 & 8.0 & 0.938 \\
\hline
\end{tabular}

such that $\operatorname{Pr}\{V(|x\rangle,|\xi\rangle)=1\} \geq 1-\epsilon$; and (ii) for all $x \in L_{n}$ and $|\xi\rangle \in \mathcal{H}^{\pi(|x|)}, \operatorname{Pr}\{V(|x\rangle,|\xi\rangle)=1\} \leq \epsilon$. Here $\operatorname{Pr}\{V(|x\rangle,|\xi\rangle)=1\}$ is the probability that $V$ concludes $x \in L_{y}$ when the quantum witness is $|\xi\rangle$.

Informally, if $x$ is a Yes (No) instance, there exists a (no) quantum witness $|\xi\rangle$ which causes $V$ to correctly (mistakenly) conclude $x \in L_{y}$ with probability at least $1-\epsilon$ (greater than $\epsilon$ ).

A promise problem is $Q M A$-Complete if it belongs to $Q M A$ and all problems in $Q M A$ are polynomially reducible to it. It has been shown [15, 16] that $k$-Local Hamiltonian is $Q M A$-Complete for $k \geq 2$.

Definition 2 ( $k$-Local Hamiltonian) Consider an $L$ qubit Hamiltonian $H=\sum_{j=1}^{r} H_{j}$, where $r=\operatorname{poly}(L)$; and each term $H_{j}$ acts on at most $k$ qubits (k-local); has operator norm $\left\|H_{j}\right\| \leq \operatorname{poly}(L)$; and matrix elements specified by poly $(L)$ bits. Finally, two constants $a<b$ are specifed. The Hamiltonian $H$ is a Yes instance if its groundstate energy $E_{g s}<a$, and a No instance if $E_{g s}>b$. The problem is, given a k-local Hamiltonian $H$, determine whether $H$ is a Yes or a No instance.

Our Ramsey number AQE algorithm leads naturally to an example of $t$-Local Hamiltonian which we call RAMSEY. We have seen that the Ramsey problem Hamiltonian $H_{P}^{N m n}$ is a $t$-local Hamiltonian; is a sum of a polynomial number of terms $H_{j}=H_{\alpha}$ or $\bar{H}_{\alpha}$; and each $H_{j}$ satisfies the polynomial bounds specified in Definition 2. Suitable choices for the constants $a$ and $b$ are $0.01<a<0.1$ and $b=1-a$. Yes instances of RAMSEY then correspond to $N<R(m, n)$ since $E_{g s}=0<a$, and No instances to $N \geq R(m, n)$ where $E_{g s} \geq 1>b$. It is possible to carry over the proof that $k$-Local Hamiltonian is in $Q M A$ [15] to show that RAMSEY is also in $Q M A$.

For an AQE algorithm with non-degenerate groundstate (GS), the runtime is largely determined [8] by the minimum energy gap $\Delta=\min _{t}\left\{E_{1}(t)-E_{0}(t)\right\}$. This connection fails for the Ramsey algorithm when $N=R(m, n)$ as the GS becomes degenerate during its execution and so $\Delta$ vanishes. Determining how the runtime scales when $\Delta=0$ (as with the Ramsey algorithm) is an open problem in adiabatic quantum computing.

In this paper we have presented a quantum algorithm that calculates two-color Ramsey numbers $R(m, n)$; nu- merically simulated the algorithm and shown it correctly determined small Ramsey numbers; discussed its experimental implementation; and shown that Ramsey number computation is in the quantum complexity class $Q M A$.

We thank W. G. Macready, P. Young, S. Jordan, and M. J. O'Hara for valuable comments, and F. G. thanks T. Howell III for continued support.

[1] R. L. Graham, B. L. Rothschild, J. H. Spencer, Ramsey Theory (Wiley, New York, 1990).

[2] J. Nešetruil, in Handbook of Combinatorics, eds. R. L. Graham, M. Grötschel, L. Lovász (Elsevier, New York, 1995), Vol. 2, p. 1331.

[3] B. Bollobás, Modern Graph Theory (Springer, New York, 1998).

[4] J. Paris, L. Harrington, in Handbook of Mathematical Logic, ed. J. Bairwise (North Holland, Amsterdam, 1977), p. 1133.

[5] H. Furstenberg, Recurrence in Ergodic Theory and Combinatorial Number Theory (Princeton, Princeton, NJ, 1981).

[6] V. Rosta, Electronic J. Combinatorics (2004), Dynamical Survey DS 13.

[7] J. Ketonen and R. Solovay, Ann. Math. 113, 267 (1981).

[8] E. Farhi, J. Goldstone, S. Gutmann, M. Sipser, 2000, available at arXiv.org quant-ph/0001106 v1.

[9] J. Spencer, Ten Lectures on the Probabilistic Method, 2nd ed. (SIAM, Philadelphia, PA, 1994).

[10] E. Farhi, J. Goldstone, S. Gutmann, J. Lapan, A. Lundgren, D. Preda, Science 292, 472 (2001).

[11] A. W. Goodman, Amer. Math. Monthly 66, 778 (1959).

[12] L. H. Clark, J. Graph Theory 16, 451 (1992).

[13] F. Gaitan, Int. J. Quantum Info. 4, 843 (2006).

[14] F. Gaitan, Complexity 14, issue 6, 21 (2009).

[15] A. Y. Kitaev, A. H. Shen, M. N. Vyalyi, Classical and Quantum Computation (American Mathematical Society, Providence, RI, 2000), Section 14.

[16] J. Kempe, A. Kitaev, O. Regev, SIAM J. Comput. 35, 1070 (2006).

[17] R. Oliveira, B. M. Terhal, Quant. Info. Comp. 8, 900 (2008).

[18] S. P. Jordan, E. Farhi, Phys. Rev. A 77, 062329 (2008).

[19] M. Steffen, W. van Dam, T. Hogg, G. Breyta, I. Chuang, Phys. Rev. Lett. 90, 067903 (2003).

[20] X. Peng et al., Phys. Rev. Lett. 101, 220405 (2008).

[21] R. Harris et al., Phys. Rev. B 82, 024511 (2010). 